\title{
Dietary Fatty Acids and Vitamin B3: An Effective Treatment Strategy for the Metabolic Syndrome?
}

Sergio Montserrat-de la Paz, Sergio Lopez, Almudena Ortega-Gomez, Lourdes M Varela, Rocio Abia, Francisco JG Muriana and Beatriz Bermudez*

*Corresponding author: Beatriz Bermudez, Laboratory of Cellular and Molecular Nutrition, Instituto de la Grasa (CSIC), 41012 Seville, Spain, E-mail: beabermudez@ig.csic.es 


\section{Abstract}

The metabolic syndrome (MS) may be defined as the constellation of cardiovascular disease (CVD) risk factors that comprises obesity, type 2 diabetes, dyslipidemia, and hypertension. Recent evidences suggest that, primarily due to its high monounsaturated fatty acids (MUFAs) content, olive oil and omega-3 polyunsaturated fatty acids (PUFAs) could be useful as a dietary approach for MS management, with relevance in the postprandial state. Vitamin $\mathrm{B} 3$, as a major substrate for nicotinamide phosphoribosyltransferase (NAMPT), also constitutes a nutritional intervention strategy for the treatment of MS. NAMPT has been shown to exert activities of central importance to cellular energetics and innate immunity. Within the cell, NAMPT is the rate-limiting step in a salvage pathway of nicotinamide adenine dinucleotide (NAD+) biosynthesis. NAMPT has been shown to correlate with triglycerides in the fasting plasma, and a potential regulatory role for fatty acids on NAMPT expression has been proposed. Whether different dietary fatty acids, including olive oil as a source of MUFA, play a role in NAMPT excursions and in the NAMPT-dependent regulation of glucose and lipid metabolism and inflammation states remains to be solved. In general, the mechanisms that alter NAD + metabolism probably include multiple processes, but the understandings of these mechanisms are currently very unclear and a considerable effort in this area is required before we know how changes in NAD+ metabolism influence physiology of glucose and lipid metabolism and how NAD+ metabolism might be manipulated for healing benefit by specific dietary fatty acids as a therapeutic treatment for MS.

Keywords: Metabolic syndrome, cardiovascular diseases, dietary oleic acid, omega-3 PUFAs, niacin, NAMPT, NAD+ 


\section{Introduction}

Cardiovascular disease (CVD) is the first cause of death worldwide, with type 2 diabetes (T2D) making up about $90 \%$ of the cases [1,2]. Incorporating a cluster of metabolic abnormalities, the metabolic syndrome (MS) was advocated by several organizations as a major predictor of CVD and T2D [3-6]. MS is characterized by four major traits: increased abdominal fat, hypertension, hyperglycemia, and dyslipidemia [7]. Lacks of habitual physical activity combined with diet contribute to increase the risk of CVD, and the development of MS in particular. The core components of the dyslipidemia in the MS, which most likely initiate CVD, are the "lipid triad" of high plasma triglycerides (TG), low levels of high-density lipoproteins (HDL), and a preponderance of small, dense low-density lipoproteins (LDL) at fasting [8]. Abnormally elevated postprandial (non-fasting) TG levels are also recognized as an important component for atherosclerosis, as first suggested by Zilversmit [9]. More recently, several studies have described abnormalities during the postprandial state in patients with coronary artery disease (CAD) [10] and have shown that non-fasting TG levels are independent predictors of CAD in multivariate analysis [11], even after adjustment for fasting TG or HDL levels in normolipidemic men. Exacerbated nonfasting TG levels are often found in insulin-resistant subjects, denoting that hyperinsulinemia and/or decreased insulin sensitivity are involved in altered postprandial metabolism of dietary fats [12].

One of the current global recommendations to counteract disability and premature death resulting from CVD is to decrease the consumption of energy-dense diets, including high-fat foods enriched in saturated fatty acids (SFAs). The most effective replacement for SFAs in terms of risk factor outcomes for CVD is the monounsaturated fatty acids (MUFAs), and the polyunsaturated fatty acids (PUFAs) but 
in much less importance [13]. In accordance, recent studies have demonstrated that olive oil, which is the only natural and most relevant source of MUFAs in the diet, when compared with butter, can postprandially limit TG excursions and buffer the pancreatic $\beta$-cell hyperactivity and peripheral insulin intolerance in subjects with normal [14] and high fasting [15] TG levels.

Dietary fatty acids have received considerable attention for their ability to regulate inflammatory gene expression and secretion. It has been proposed that dietary fatty acids affect insulin resistance and inflammatory processes through the modulation of transcription factors such as $\mathrm{NF \kappa B}$ and peroxisome proliferator-activated receptor gamma $(\operatorname{PPAR} \gamma)[16,17]$.

There is general agreement that increasing dietary SFA intake, especially in overweight or obese individuals, is associated with raised inflammatory markers [18], predominately by activating the toll-like receptor 4 (TLR4) pathway. TLR4 is expressed in both subcutaneous (SAT) and visceral (VAT) adipose tissues. SFAs serve as ligands for TLR4, inducing inflammatory responses in both adipocytes and macrophages through an increase of adipocytokine gene expression and production $[19,20]$. Moreover, SFA is associated with NFkB activation [21] which is a fundamental component underlying chronic inflammatory diseases such as atherosclerosis, rheumatoid arthritis, and MS [22]. Conversely, unsaturated fatty acid have well known anti-inflammatory effects, which range from the inhibition of the lipoxygenase and cyclooxygenase pathways, inhibition of TLR4 signaling, and PPAR $\gamma$ activation $[23,24]$. Thus, MUFAs can reproduce a number of the anti-inflammatory effects of TLR4 or induce TNF- $\alpha$ inhibition [25], which suggest that dietary MUFAs constitute an attractive nutritional approach for the treatment of MS.

PPAR $\gamma$ is a fatty acid sensor that adapts $\beta$-cell mitochondrial function [26]. 
Unsaturated fatty acids enhance mitochondrial oxidation levels in insulin-secreting cells, which protects against $\beta$-cell dysfunction [27]. In line it has been demonstrated that NAMPT (also known as visfatin or PBEF) improves insulin sensitivity and exerts its hypocholesterolemic effects at least partially through upregulation of the tyrosine phosphorylation of IRS-1 protein and the mRNA levels of PPAR $\gamma$ and SREBP-2 [28].

NAMPT is considered as a multifunctional adipokine involved in the regulation of different pathophysiological conditions. NAMPT is the rate-limiting enzyme in the $\mathrm{NAD}+$ biosynthetic pathway starting from nicotinamide and essential when the cellular $\mathrm{NAD}+$ pool is depleted, for example through poly-ADP-ribosylation during DNA repair and NAD+-dependent protein deacetylase activity. Two forms of NAMPT exist (Figure 1), intracellular (iNAMPT) and extracellular (eNAMPT). While the function of iNAMPT as a NAD+ biosynthetic enzyme is well established, the physiological role of eNAMPT is still a matter of debate [29]. eNAMPT is positively secreted through a nonclassical secretory pathway by fully differentiated human adipocytes and hepatocytes. It has been suggested that eNAMPT exhibits robust, even higher NAD+ biosynthetic activity compared to iNAMPT and that NAD+ biosynthesis mediated by iNAMPT and eNAMPT plays a critical role in the regulation of glucose-stimulated insulin secretion in pancreatic $\beta$-cells.

The observation that NAMPT has insulin-mimetic functions and is involved in the modulation of inflammation has raised the hypothesis that a dysregulation of the activity of this molecule may contribute to T2D and MS. Many research papers have been published on this topic, with controversial findings. Fasting NAMPT levels were found to be elevated in T2D patients, in women with gestational diabetes, and in extremely obese individuals (BMI $>40$ ), whereas fasting NAMPT levels were reduced by weight loss [30]. By contrast, other studies have failed to detect any correlation 
between fasting NAMPT levels and body fat or insulin sensitivity [31]. In addition, NAMPT expression was not altered in a rodent model of the MS as compared with wild-type animals. Thus, the role of NAMPT in insulin resistance and diabetes is largely unknown and deserves further examination.

Niacin (NA) or Vitamin B3 is a major substrate for NAMPT. In doses large enough to produce pharmacological effects, NA is a potent lipid-modifying agent with a broad spectrum of effects, including those aimed at attenuating the risks associated with low HDL, high LDL and TG levels in fasting plasma [32]. It is interesting to note that fasting TG levels strongly and positively correlated, and represented an independent factor associated, with NAMPT levels in healthy subjects and patients with obesity and/or T2D [30]. Moreover, fasting TG levels positively correlated with NAMPT mRNA expression in the VAT of obese subjects [31]. In contrast, there is also evidence of positive correlation between NAMPT and HDL levels, and of negative correlation between NAMPT and TG levels in the fasting state in a cohort of subjects with genetic predisposition for dyslipidemia [33], suggesting that NAMPT may be an indicator of beneficial lipid profile. In support of this notion, oral administration of NA significantly increased cellular NAD+ levels and had the ability to reduce fasting TG and LDL, whereas raising HDL [34]. These findings raise questions regarding of whether NA administration and the type of dietary fatty acids might be harmful or helpful NAMPT modulators to influence lipid homeostasis. At this stage, it is possible to argue that exogenous NA and the type of fatty acids in plasma TG may regulate NAMPT metabolism and function. This assumption substantiates the thought that exogenous TG in postprandial TRL may evoke pro- or anti-inflammatory signals in a fatty aciddependent fashion. Interestingly, a regulatory role for individual fatty acids, such as MUFAs (oleic acid), SFAs (palmitic acid), and omega-3 PUFAs (eicosapentaenoic 
acid) on NAMPT gene expression has been described in 3T3-L1 murine adipocytes and monocytes $[35,36]$. In obese subjects, a recent cross-sectional study of associations between nutrient intake and fasting NAMPT levels has also demonstrated that MUFA intake, in the adjusted multivariate analysis, was the only independent predictor of fasting NAMPT levels [37].

Despite this considerable amount of data, whether different dietary fatty acids, including olive oil as a source of MUFAs and omega-3 PUFAs, play a role in NAMPT excursions and in the NAMPT-dependent regulation of glucose and lipid metabolism and inflammation in the fed and postprandial states remains to be solved.

In general, the mechanisms that alter NAD+ metabolism probably include multiple processes, but the understandings of these mechanisms are currently very unclear and a considerable effort in this area is required before we know how changes in $\mathrm{NAD}+$ metabolism influence physiology of glucose and lipid metabolism and how $\mathrm{NAD}+$ metabolism might be manipulated for healing benefit by specific dietary fatty acids.

\section{References}

1. WHO (2011) Global atlas on cardiovascular disease prevention and control: policies, strategies and interventions..

2. WHO (2011) Diabetes: Fact sheet no. 312.

3. Alberti KG, Eckel RH, Grundy SM, Zimmet PZ, Cleeman JI, et al. (2009) International Diabetes Federation Task Force on Epidemiology and Prevention; Hational Heart, Lung, and Blood Institute; American Heart Association; World Heart Federation; International Atherosclerosis Society; International Association for the Study of Obesity. Harmonizing the metabolic syndrome: a joint interim statement of the 
International Diabetes Federation Task Force on Epidemiology and Prevention; National Heart, Lung, and Blood Institute; American Heart Association; World Heart Federation; International Atherosclerosis Society; and International Association for the Study of Obesity. Circulation120: 1640-1645.

4. Mancia G, Bombelli M, Facchetti R, Casati A, Ronchi I, et al. (2010)Impact of different definitions of the metabolic syndrome on the prevalence of organ damage, cardiometabolic risk and cardiovascular events. J Hypertens28: 999-1006.

5. Martínez-Larrad MT, Lorenzo C, González-Villalpando C, Gabriel R, Haffner SM, et al. (2012) Associations between surrogate measures of insulin resistance and waist circumference, cardiovascular risk and the metabolic syndrome across Hispanic and non-Hispanic white populations. Diabet Med29: 1390-1394.

6. Duhper S, Buddhe S, Patel S (2013) Managing cardiovascular risk in overweight children and adolescents. Pediatr Drugs15: 181-190.

7. Di Lorenzo C, Dell'agli M, Colombo E, Sangiovanni E, Restani P (2013) Metabolic syndrome and inflammation: a critical review of in vitro and clinical approaches for benefit assessment of plant food supplements. Evid Based Complement Alternat Medhttp://dx.doi.org/10.1158/2013/782461

8. Nakajima K (2010) Pharmacotherapy of mixed dyslipidemia in the metabolic syndrome. Curr Clin Pharmacol5: 133-139.

9. Zilversmit DB (1979) Atherogenesis: a postprandial phenomenon. Circulation 60: $473-485$.

10. Nakamura T, Obata JE, Takano H, Kawabata K, Sano K, et al. (2009) High serum levels of remnant lipoproteins predict ischemic stroke in patients with metabolic syndrome and mild carotid atherosclerosis. Atherosclerosis 202 234-240.

11. Nordestgaard BG, Langsted A, Freiberg JJ (2009) Nonfasting hyperlipidemia and 
cardiovascular disease. Curr Drug Targets 10 328-335.

12. Enkhmaa B, Ozturk Z, Anuurad E, Berglund L (2010) Postprandial lipoproteins and cardiovascular disease risk in diabetes mellitus. Curr Diab Rep 10 61-69.

13. Ortega A, Varela LM, Bermudez B, Lopez S, Abia R, et al. (2012) Dietary fatty acids linking postprandial metabolic response and chronic diseases. Food Funct 3: 2227.

14. López S, Bermúdez B, Pacheco YM, Villar J, Abia R, et al. (2008) Distinctive postprandial modulation of beta cell function and insulin sensitivity by dietary fats: monounsaturated compared with saturated fatty acids. Am J Clin Nutr 88 638-644.

15. Lopez S, Bermudez B, Ortega A, Varela LM, Pacheco YM, et al. (2011) Effects of meals rich in either monounsaturated or saturated fat on lipid concentrations and on insulin secretion and action in subjects with high fasting triglyceride concentrations. Am J Clin Nutr 93: 494-499.

16. Siriwardhana N, Kalupahana NS, Cekanova M, LeMieux M, Greer B, et al. (2013) Modulation of adipose tissue inflammation by bioactive food compounds. J Nutr Biochem24: 613-623.

17. Van den Berghe W, Vermeulen L, Delerive P, De Bosscher K, Staels B, et al.

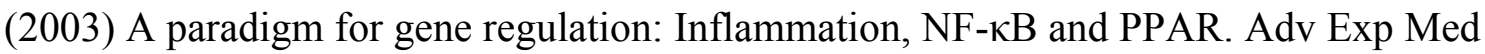
Biol 544: 181-196.

18. Calder PC, Ahluwalia N, Brouns F, Buetler T, Clement K, et al. (2011) Dietary factors and low-grade inflammation in relation to overweight and obesity. $\mathrm{Br} \mathrm{J}$ Nutr 106: S5-S78.

19. Fessler MB, Rudel LL, Brown JM (2009) Toll-like receptor signaling links dietary fatty acids to the metabolic syndrome. Curr Opin Lipidol 20: 379-385.

20. Sears B, Ricordi C (2012) Role of fatty acids and polyphenols in inflammatory gene 
transcription and their impact on obesity, metabolic syndrome and diabetes. Eur Rev Med Pharmacol Sci 16: 1137-1154.

21. Suganami T, Ogawa Y (2010) Adipose tissue macrophages: their role in adipose tissue remodeling. J Leukoc Biol 88: 33-39.

22. Killeen MJ, Linder M, Pontoniere P, Crea R (2013) NF- $\kappa B$ signaling and chronic inflammatory diseases: exploring the potential of natural products to drive new therapeutic opportunities. Drug Discov Today 19: 373-378.

23. Mihály J, Gericke J, Törőcsik D, Gáspár K, Szegedi A, et al. (2013) Reduced lipoxygenase and cyclooxygenase mediated signaling in PBMC of atopic dermatitis patients. Prostaglandins Other Lipid Mediat 18, S1098-8823(13)00021-X.

24. Norris PC, Dennis EA (2012) Omega-3 fatty acids cause dramatic changes in TLR4 and purinergic eicosanoid signaling. Proc Natl Acad Sci USA 109: 8517-8522.

25. Sánchez-Fidalgo S, Cárdeno A, Sánchez-Hidalgo M, Aparicio-Soto M, et al. (2013) Dietary extra virgin olive oil polyphenols supplementation modulates DSS-induced chronic colitis in mice. J Nutr Biochem 24: 1401-1413.

26. Poulsen Ll, Siersbæk M, Mandrup S (2012) PPARs: fatty acid sensors controlling metabolism. Semin Cell Dev Biol 23: 631-639.

27. García-Ruiz I, Solís-Muñoz P, Fernández-Moreira D, Muñoz-Yagüe T, SolísHerruzo JA (2013) Pioglitazone leads to an inactivation and disassembly of complex I of the mitochondrial respiratory chain. BMC Biol11: 88 .

28. Sun Q, Li L, Li R, Yang M, Liu H, et al. (2009) Overexpression of visfatin/PBEF/Nampt alters whole-body insulin sensitivity and lipid profile in rats. Ann Med 41: 311-320.

29. Garten A, Petzold S, Korner A, Imai S, Kiess W (2009) Nampt: linking NAD biology, metabolism and cancer. Trends Endocrinol Metab 20: 130-138. 
30. Esteghamati A, Alamdari A, Zandieh A, Elahi S, Khalilzadeh O, et al. (2010) Serum visfatin is associated with type 2 diabetes mellitus independent of insulin resistance and obesity.Diabetes Res Clin Pract 91: 154-158.

31. Chang YC, Chang TJ, Lee WJ, Chuang LM (2010) The relationship of visfatin/preB-cell colony-enhancing factor/nicotinamide phosphoribosyltransferase in adipose tissue with inflammation, insulin resistance, and plasma lipids. Metabolism 59: 93-99.

32. Nelson RH, Vlazny D, Smailovic A, Miles JM (2012) Intravenous niacin acutely improves the efficiency of dietary fat storage in lean and obese humans. Diabetes 61: $3172-3175$

33. Wang P, van Greevenbroek MM, Bouwman FG, Brouwers MC, van der Kallen CJ, et al. (2007) The circulating PBEF/NAMPT/visfatin level is associated with a beneficial blood lipid profile. Pflugers Arch 454: 971-976.

34. Schachter M (2005) Strategies for modifying high-density lipoprotein cholesterol: a role for nicotinic acid. Cardiovasc Drugs Ther 19: 415-422.

35. Wen Y, Wang $\mathrm{HW}, \mathrm{Wu}$ J, Lu HL, Hu XF, et al. (2006) Effects of fatty acid regulation on visfatin gene expression in adipocytes. Chin Med J 119: 1701-1708.

36. de Kreutzenberg SV, Ceolotto G, Papparella I, Bortoluzzi A, Semplicini A, et al. (2010) Downregulation of the longevity associated protein sirtuin 1 in insulin resistance and metabolic syndrome: potential biochemical mechanisms. Diabetes 59: 1006-1015. 37. de Luis DA, Aller R, Gonzalez Sagrado M, Conde R, Izaola O, et al. (2010) Serum visfatin concentrations are related to dietary intake in obese patients. Ann Nutr Metab 57: $265-270$ 
Legend to Figure

Figure 1. Model of putative modes of action for NAMPT to affect cell metabolism adaptaed from Garten et al. [29]. NAMPT functions as an intra- and extracellular NAD + biosynthetic enzyme. NAMPT catalyzes the formation of nicotinamide mononucleotide $(\mathrm{NMN})$ from nicotinamide (NAM). NMN is subsequently converted to NAD + by three organelle-specific isoforms of nicotinamide mononucleotide adenylyltransferase (Nmnat1-3). Intracellularly, NAMPT has been shown to be located in different cellular compartments. It affects the function of NAD+-degrading enzymes by raising cellular $\mathrm{NAD}+$ levels and consequently influences the regulation of metabolism and stress resistance through sirtuins (Sirt1-7) and other NAD+-consuming enzymes, such as poly(ADP-ribose)polymerase-1 (PARP-1). The NAD+ metabolism of (a) mitochondria and (b) nucleus is possibly influenced by transport of NAD+ metabolites from and to the cytoplasm. (c) Extracellularly, NAMPT produces nicotinamide mononucleotide (NMN), which might act in an autocrine/paracrine fashion and/or be transported to other target tissues, where it acts on glucose-stimulated insulin secretion in pancreatic $\beta$-cells and potentially elicits other biological responses. (d) Possibly, NAMPT also functions as a cytokine by binding to and activating an unidentified receptor. 


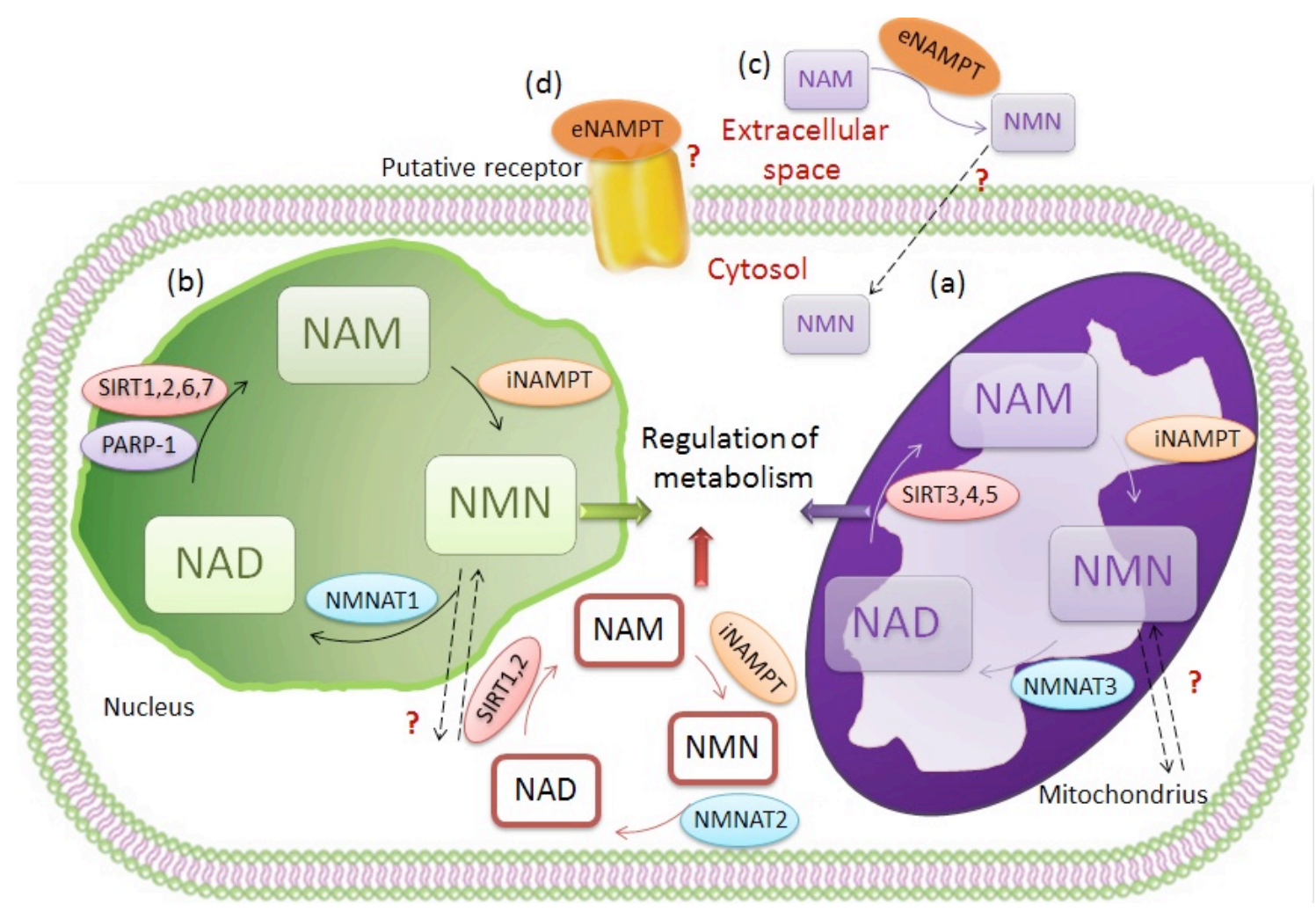

